\title{
ON SINGULAR POINTS IN THE APPROXIMATE DEVELOPMENT \\ OF THE PERTURBATIVE FUNCTION
}

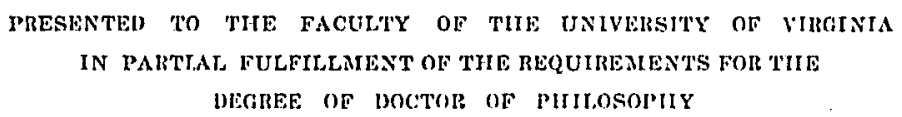

BY

FRANK WALKER REED

Reprinted from the

TRANSACTIONS OF THE AMERICAN MATHEMATICAL SOCIETY

Vol. X., No. 4, pp. 485-509

New York, Ootober, 1909 


\title{
ON SINGULAR POINTS IN THE APPROXIMATE DEVELOPMENT
}

\author{
OF THE PERTURBATIVE FUNCTION*
}

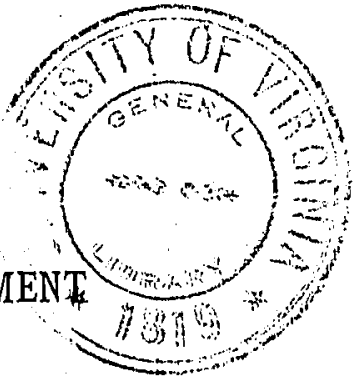

BY

\section{FRANK WALKER REED}

\section{§ 1. INTRODUCTION.}

A method of computing approximately the coefficients of those terms of high order in the perturbative function which derive their inportance from the presence in them of a small divisor has been set forth by Poincart. As applications of the method to the problem of three bodies, Porncare $\uparrow$ has considered the case of coplanar orbits, one elliptic and one circular, and the case of coplanar elliptic orbits, both ecentricities being small. The latter case has been developed in detail by Coculesco. $\neq \mathrm{H}_{\text {AMY }} \S$ has discussed the cases of an elliptic enclosing a circular orbit and a circular enclosing an elliptic orbit, the mutual inclination being small, and the case of circular orbits with any inclination. The latter of these was discussed anew by Frraud \| together with the case of an elliptic enclosing a coplanar circular orbit, and the case of a circular enclosing a coplanar elliptic orbit.

Owing to the high degree of the algebraic equations which define the singular points upon which the approximate values of the coefficients depend, it is found necessary, in the general case, to resort to numerical examples. This paper is devoted to a discussion of these algebraic equations in certain cases more general than those so far considered, and to an extension of the criterion of admissibility as employed by PorNCARs. The singular points in the most general case and in an important particular case are found and classified. The results in the cases studied by Porncare and Coculesco are given first because of their usefulness in treating the more general problem. Before proceeding to

\footnotetext{
* Presented to the Society (Princeton) September 13, 1909.

† Poincarí. Les Méthodes Nouvelles de la MÍ́canique Céleste, vol. 1, Chap. VI.

† Coculasco. Sur les expressions approchées des termes d'ordre éle tó dans le développement de la fonction perturbatrice, Journal de Mathématiques, ser. 4, vol. 1 (1895).

\& Harry. Sur les ineqalités d'ordre élevé, Bulletin Astronomique, vol. 10 (1893). Sur le développement approché de la fonction perturbatrice, Journal de Mathématiques, ser. 4, vol. 10 (1894); ser. 5, vol. 2 (1896).

| Fóraud. Sur la valeur approclié des corfficients d'ordre élevé dans le développement en series, Annales de l'Observatoire de Bordeaux, vol. 7.
} 
this discussion, it is necessary to set forth briefly the principles of Poincare's method, but in doing so the general equations are derived at once.

It is required to develop a certain function $F_{1}^{0}$ in terms of the mean anomalies $l$ and $l^{\prime}$ in the form

$$
F_{1}^{\prime \prime}=\sum_{m_{1}=0}^{\infty} \sum_{m_{2}=0}^{\infty} A_{m_{1} m_{2}} e^{i\left(m_{1} l+m_{2} l^{\prime \prime}\right)}, \quad i=\sqrt{-1}, \quad \text { and } \quad m_{1}, m_{2} \text { integers. }
$$

The coefficients $A_{m_{1} m_{2}}$ are given by the integral

where

$$
A_{m t_{3} m_{n}}=\frac{1}{2 i \pi} \int_{|=|=1} z^{-n-1} \Phi(z) d z
$$

and

$$
\Phi(z)=\frac{1}{2 i \pi} \int_{|t|=1} F(z, t) d t
$$

$$
F(z, t)=F_{1}^{\prime \prime} t^{u t-b c-1} z^{-\frac{i}{n}} \text {. }
$$

The new variables, $t$ and $z$, have been introduced by the transformation

$$
e^{i l}=t^{\prime \prime}, \quad e^{i l \prime}=t^{-i} z^{\frac{1}{n}},
$$

and the new constants by the relations

$$
m_{1}=a n+b, \quad m_{2}=c n+d
$$

where $a, b, c, d$ are finite integers, small relative to $n$, and $a$ and $c$ are relatively prime. Now if $\Phi(z)$ is expanded as a Laurent series

$$
\Phi(z)=\sum_{n=0}^{\infty} a_{n} z^{n}+\sum_{n=0}^{\infty} a_{-n} z^{-n}
$$

and the result substituted in the expression defining $A_{m_{1} m_{2}}$, we get

$$
A_{m_{1} m_{2}}=a_{n} .
$$

A theorem by DARBoux * can be employed to obtain approximate values of $a_{n}$, and consequently of $A_{m_{1} m_{2}}$, for large values of $n$ without computing the intervening coefficients.

The initial contonr of integration, $|t|=1$, may be deformed in any manner without affecting the value of the integral $\Phi(z)$ provided no singular point of $F(z, t)$, considered as a function of $t$ with $\approx$ as a parameter, is encountered. By varying $z$ continuously the singular points of $F(z, t)$ are displaced continuously. Singular points of $\Phi(z)$ are those values of $z$ for which two singular

* Darizoux. Sur l'approrimution des fonctions de tress-grands nombres, Journal de Mathematiques, ser. 3 , vol. 4 (1878). 
points of $F(z, t)$, considered as a function of $t$, come into coincidence. If the points were initially, that is for $|z|=1$, one inside and the other outside the contour $|t|=1$, then the singular point $z$ of $\Phi(z)$ is admissible; for, $\Phi(z)$ ceasing to be holomorphic, the theorem of Darboux is applicable.

The principal part of the perturbative function * is

where

$$
F_{1}^{0}=L^{-2} P^{\prime-1}
$$

$$
\begin{gathered}
P^{\prime}=(\xi-\beta \eta)\left(\xi_{0}-\beta_{0} \eta_{0}\right)+\frac{1}{2}(\cos J-1)\left[\gamma \xi \eta+\gamma_{0} \xi_{0} \eta_{0}-\left(\beta_{0} \xi \eta_{0}+\beta \xi_{0} \eta\right)\right] \\
\beta=L^{\prime 2} L^{-2} e^{i\left(\overline{\omega^{\prime}}-\bar{\omega}\right)}, \quad \beta_{0}=L^{\prime 2} L^{-2} e^{-i\left(\bar{\omega}^{\prime}-\bar{\omega}\right)} \\
\gamma=L^{\prime 2} L^{-2} e^{i\left(\overline{\omega^{\prime}}+\bar{\omega}\right)}, \quad \gamma_{0}=L^{\prime 2} L^{-2} e^{-i\left(\bar{\omega}^{\prime}+\bar{\omega}\right)} .
\end{gathered}
$$

$L^{2}, \sin \phi, l, u, \bar{\omega}$ represent respectively the major axis, the eccentricity, the mean anomaly, the eccentric anomaly, and the longitude of perihelion of a planet $B$ referred to the line of nodes and the center of gravity, $D$, of $A$ and $B$ in a system of three bodies $A, B, C . L^{\prime 2}$, sin $\phi^{\prime}$, etc. are the elements of $C$ referred to $D$ and the lines of nodes. $J$ is the inclination. $\xi, \eta$ are defined by

$$
\begin{aligned}
& \xi=\cos u-\sin \phi+i \cos \phi \sin u, \\
& \eta=\cos u^{\prime}-\sin \phi^{\prime}+i \cos \phi^{\prime} \sin u^{\prime},
\end{aligned}
$$

and $\xi_{0}, \eta_{0}$ are their conjugates. With this notation the coördinates of $B$ and $C$ respectively are given by the real and imaginary parts of

respectively.

$$
\xi L^{2} e^{i \bar{\omega}}, \quad \eta L^{\prime 2} e^{\overline{\omega_{\omega}^{\prime}}},
$$

The singular points of $F^{\prime}(z, t)$ with which we are concerned are the same as those of $F_{l}^{0}$. These are $(a)$ the points for which $u$ and $u^{\prime}$ as functions of $l$ and $l^{\prime}$ cease to be uniform, given by

$$
\frac{\partial l}{\partial u}=1-\sin \phi \cos u=0, \quad \frac{\partial l^{\prime}}{\partial u^{\prime}}=1-\sin \phi^{\prime} \cos u^{\prime}=0,
$$

and $(b)$ the singularities of $F_{1}^{0}$, given by

Making the substitutions

$$
P^{\prime}=0 \text {. }
$$

$$
\begin{aligned}
x=e^{i u}, \quad y=e^{i u^{\prime}}, & j=\frac{1}{2}(\cos J-1), \\
\tan \frac{\phi}{2}=\tau, & 1+\tau^{2}=\sigma, \\
\tan \frac{\phi^{\prime}}{2}=\tau^{\prime}, & 1+{\tau^{\prime}}^{2}=\sigma^{\prime},
\end{aligned}
$$

* This form of the perturbative function is due to FeraUd, loc. cit.

Trans. Am. Matlı. Snc. 32 
we get

$$
\begin{array}{ll}
\xi=\frac{(x-\tau)^{2}}{\sigma x}, & \xi_{0}=\frac{(1-x \tau)^{2}}{\sigma x} \\
\eta=\frac{\left(y-\tau^{\prime}\right)^{2}}{\sigma^{\prime} y}, & \eta_{0}=\frac{\left(1-y \tau^{\prime}\right)^{2}}{\sigma^{\prime} y} .
\end{array}
$$

Then the equations defining the singularities become

$$
\begin{aligned}
& (x-\tau)(1-x \tau)=0, \\
& \left(y-\tau^{\prime}\right)\left(1-y \tau^{\prime}\right)=0,
\end{aligned}
$$

$$
P=\left(\sigma \sigma^{\prime} x y\right)^{2} P^{\prime}=\left[\sigma^{\prime} y(x-\tau)^{2}-\beta \sigma x\left(y-\tau^{\prime}\right)^{2}\right]
$$

$$
\begin{aligned}
\times & {\left[\sigma^{\prime} y(1-x \tau)^{2}-\beta_{0} \sigma x\left(1-y \tau^{\prime}\right)^{2}\right]+j \sigma \sigma^{\prime} x y\left[\gamma(x-\tau)^{2}\left(y-\tau^{\prime}\right)^{2}\right.} \\
& \left.+\gamma_{0}(1-x \tau)^{2}\left(1-y \tau^{\prime}\right)^{2}-\beta_{0}(x-\tau)^{2}\left(1-y \tau^{\prime}\right)^{2}-\beta(1-x \tau)^{2}\left(y-\tau^{\prime}\right)^{2}\right]=0 .
\end{aligned}
$$

Singular points of the first species of $\Phi(z)$ are given by the intersections of the curves $(1),(2),(3)$. Singular points of the second species are those satisfying the condition for double roots of (1), (2), or (3), any one of which may be written in the general form in the original variables

$$
f(z, t)=0 \text {. }
$$

The condition for a multiple root of $f(z, t)$ is

$$
f=0, \quad \frac{\partial f}{\partial t}=\frac{c \sigma x^{2}}{(x-\tau)(1-x \tau)} \frac{\partial f}{\partial x}-\frac{a \sigma^{\prime} y^{2}}{\left(y-\tau^{\prime}\right)\left(1-y \tau^{\prime}\right)} \frac{\partial f}{\partial y}=0 .
$$

Equations (1) and (2) will not give rise to points of the second species.

Consider equation (3), which may be written

where

$$
P(x, y)=B C+j \sigma \sigma^{\prime} x y A
$$

$$
\begin{aligned}
& A=\gamma(x-\tau)^{2}\left(y-\tau^{\prime}\right)^{2}+\gamma_{0}(1-x \tau)^{2}\left(1-y \tau^{\prime}\right)^{2} \\
& -\beta_{0}(x-\tau)^{2}\left(1-y \tau^{\prime}\right)^{2}-\beta(1-x \tau)\left(y-\tau^{\prime}\right)^{2}, \\
& B=\sigma^{\prime} y(x-\tau)^{2}-\beta \sigma x\left(y-\tau^{\prime}\right)^{2}, \\
& C=\sigma^{\prime} y(1-x \tau)^{2}-\beta_{0} \sigma x\left(1-y \tau^{\prime}\right)^{2} .
\end{aligned}
$$

The equation $\partial P / \partial t=0$ becomes, after reducing by means of $P=0$,

$$
R=B^{\prime} C(x-\tau)\left(y-\tau^{\prime}\right)-B C^{\prime}(1-x \tau)\left(1-y \tau^{\prime}\right)
$$




$$
\begin{aligned}
-j c \sigma x\left(y-\tau^{\prime}\right) & \left(1-y \tau^{\prime}\right)\left[A-x \frac{\partial A}{\partial x}\right] \\
& +j a \sigma^{\prime} y(x-\tau)(1-x \tau)\left[A-y \frac{\partial A}{\partial y}\right]=0,
\end{aligned}
$$

where

$$
\begin{aligned}
& B^{\prime}=c(x+\tau)\left(1-y \tau^{\prime}\right)+a \beta\left(y+\tau^{\prime}\right)(1-x \tau), \\
& C^{\prime}=c(1+x \tau)\left(y-\tau^{\prime}\right)+a \beta_{0}\left(1+y \tau^{\prime}\right)(x-\tau) .
\end{aligned}
$$

\section{$\S 2$. Coplanar Orbits.}

The equations $P=0$ and $R=0$, which determine all the singular points of the second species in the most general case, admit of important simplifications in certain particular cases. If we suppose the inclination zero, then $j=0$, and we have
(1) $(x-\tau)(1-x \tau)=0$,
(3) $B=0$,
(5) $B^{\prime}=0$,
(2) $\left(y-\tau^{\prime}\right)\left(1-y \tau^{\prime}\right)=0$,
(4) $C=0$
(6) $C^{\prime}=0$,

where (3), (4) are the factors of the reduced equation $P=0$, and (5), (6), those of $R=0$. In this case the singularities are as follows:

$B=0, x=\tau$, and $y=\tau^{\prime}$ intersect in $U, \quad C=0$, and $y=\tau^{\prime}$ intersect in $V$ and $W^{\prime}$, $C=0, x=\frac{1}{\tau^{\prime}}$ and $y=\frac{1}{\tau^{\prime}}$ intersect in $U^{\prime}, \quad B=0$, and $y=\frac{1}{\tau^{\prime}}$ intersect in $V^{\prime}$ and $W$, $C=0$, and $x=\tau$ intersect in $E$ and $Y^{\prime}, \quad x=\tau$, and $y=\frac{1}{\tau^{\prime}}$ intersect in $X$, $B=0$, and $x=\frac{1}{\tau}$ intersect in $E^{\prime}$ and $Y, \quad x=\frac{1}{\tau}$, and $y=\tau^{\prime}$ intersect in $X^{\prime}$,

$$
\begin{aligned}
& B=0 \text { and } C=0 \text { intersect in } B^{\prime}, B, R, \text { and } R^{\prime}, \\
& B=0 \text { and } B^{\prime}=0 \text { intersect in } C^{\prime}, D, T, \text { and } F, \\
& C=0 \text { and } C^{\prime}=0 \text { intersect in } T^{\prime}, D^{\prime}, C, \text { and } F^{\prime} .
\end{aligned}
$$

These singular points have been discussed by Poincare and Coculesco.

\section{\$3. The General Case.}

The main difficulty which arises in the general case is that of solving the algebraic equations. Even when $\tau, \tau^{\prime}, j$ are small, as is usually the case in nature, the approximations thus made possible do not so reduce the equations 
that they admit of literal solutions. But the problem is not without interest from the theoretical standpoint, although the method it is necessary to employ is dependent upon numerical assumptions. In fact the results to be obtained in this manner have the same generality as those which would be furnished by a literal solution.

Since for $j=0$ the equations $P=0$ and $R=0$ reduce to

$$
B=0, \quad C=0, \quad B^{\prime}=0, \quad C^{\prime}=0,
$$

it is easily seen that all the singular points for a small inclination will lie near those given in $\$ 2$. The reducible curve $P=0$ will lie near the curves $B=0$ and $C=0$, but $R=0$, being irreducible, will not lie near $B^{\prime}=0$ or $C^{\prime}=0$.

Referring to equations $(3),(4)$ of $\S 1$, and recalling the fact that $\beta, \beta_{0}$ and also $\gamma, \gamma_{0}$ are conjugate complex quantities, we can easily verify that

$$
\begin{aligned}
& A(x, y, i)=x^{2} y^{2} A\left(\frac{1}{x}, \frac{1}{y}, \frac{1}{i}\right), \\
& A-x \frac{\partial A}{\partial x}=A_{1}(x, y, i)=-x^{2} y^{2} A_{1}\left(\frac{1}{x}, \frac{1}{y}, \frac{1}{i}\right), \\
& A-y \frac{\partial A}{\partial y}=A_{2}(x, y, i)=-x^{2} y^{2} A_{2}\left(\frac{1}{x}, \frac{1}{y}, \frac{1}{i}\right) .
\end{aligned}
$$

Substituting in $P$ and $R$ above $1 / x, 1 / y, 1 / i$ for $x, y, i$, multiplying throughout by $x^{4} y^{4}$, and changing signs in the latter function, we arrive at the identical expressions for $P$ and $R$. Thus $P=0$ and $R=0$ are self-reciprocal equations in $x, y$, provided that $\beta, \beta_{0}$ and also $\gamma, \gamma_{0}$ interchange. This was to be expected, since the equations were originally in $\cos u, \cos u^{\prime}, \sin u, \sin u^{\prime}$ which involve $x, y$ reciprocally. Consequently the discussion of the singular points will be very much abridged.

The equation $P=0$ is an algebraic equation of the sixth degree; the term with $j$ as a factor is also of the sixth degree. The highest power of $x$, and also of $y$, is the fourth. The curve has the axes as double asymptotes, and has also two oblique asymptotes. The equation $R=0$ is of the seventh degree. As the highest power of $x$ or of $y$ is the fourth, the curve has three asymptotes parallel to each of the axes. Among these are the two axes. There is one oblique asymptote.

We come to consider certain restrictions which may be put upon the elements. In the planetary theory we are concerned usually with the case of orbits having small eccentricities. We proceed to apply this restriction to equations $P=0$ and $R=0$, and to note what simplifications take place. It was found in the simpler cases that the abscissas of half of the singularities were of the order $\tau$ or $\tau^{\prime}$, and that the other half were of the order $1 / \tau$ or $1 / \tau^{\prime}$. The same is true for 
the ordinates of the singular points. If $\tau, \tau^{\prime}$ are sufficiently small we have approximately

$$
\sigma=1+\tau^{2}=1, \quad \sigma^{\prime}=1+\tau^{2}=1 ;
$$

and if in addition $x$ and $y$ are small, we have approximately

$$
1-x \tau=1, \quad(1-x \tau)^{2}=1, \quad 1-y \tau^{\prime}=1, \quad\left(1-y \tau^{\prime}\right)^{2}=1 .
$$

In the expression for $A$ the coefficients of $\left(y-\tau^{\prime}\right)^{2}$ and $\left(1-y \tau^{\prime}\right)^{2}$ may be written respectively

$$
\gamma(x-\tau)^{2}-\beta(1-x \tau)^{2}, \quad \gamma_{1}(1-x \tau)^{2}-\beta_{0}(x-\tau)^{2},
$$

which become, omitting terms of the second order, $-\beta$ and $\gamma_{0}$ respectively. Making in $P=0$ the approximations indicated and arranging in powers of $y$, we get

$$
\begin{aligned}
P_{1} \equiv y^{3}[-\beta x(1+j)]+y^{2}\left[(x-\tau)^{2}+\beta \beta_{0} x^{2}+2 \beta \tau^{\prime}(1+j) x\right] & \\
& +y\left[-\beta_{0} x(x-\tau)^{2}-2 \beta \beta_{0} x^{2} \tau^{\prime}-\beta \tau^{\prime 2} x(1+j)+j \gamma_{0} x\right]+\beta \beta_{0} x^{2} \tau^{\prime 2}=0 .
\end{aligned}
$$

The equation $R=0$ likewise takes a reduced form. We have approximately

$$
A-x \frac{\partial A}{\partial x}=\gamma_{0}-\beta\left(y-\tau^{\prime}\right)^{2}, \quad A-y \frac{\partial A}{\partial y}=\gamma_{0}+\beta\left(y^{2}-\tau^{\prime 2}\right) .
$$

Introducing these expressions in $R=0$, simplifying as above, and arranging in powers of $y$, we get

$$
\begin{aligned}
R_{1} \equiv & y^{3}\left[a \beta^{\prime}(x-\tau)+c \beta^{\prime} x\right]+y^{2}\left[2 c \tau(x-\tau)-3 c \beta^{\prime} \tau^{\prime} x\right] \\
+ & y\left[-c \beta_{0} x\left(x^{2}-\tau^{2}\right)-a \beta_{0}(x-\tau)^{3}-2 c \tau^{\prime} \tau(x-\tau)-2 a \beta \beta_{0} \tau^{\prime} x(x-\tau)\right] \\
& -a \beta^{\prime} \tau^{\prime 2}(x-\tau)+3 c \beta^{\prime} \tau^{\prime 2} x-j c \gamma_{0} x+j a \gamma_{0}(x-\tau) \\
& +c \beta_{0} \tau^{\prime} x\left(x^{2}-\tau^{2}\right)+2 a \beta \beta_{10} \tau^{\prime 2} x(x-\tau)-c \beta^{\prime} \tau^{\prime 3} x+j c \gamma_{0} \tau^{\prime} x=0,
\end{aligned}
$$

where $\beta^{\prime}=\beta(1+j)$.

Thus we have a system of equations, (1), (2), $P_{1}=0, R_{1}=0$, for determining the singular points of $\Phi(z)$ which lie near the origin. It is unnecessary to discuss the geometry of the curves $P_{1}=0, R_{1}=0$. We know that in the region in which $x, y$ are of the order $\tau, \tau^{\prime}$ these represent very closely the curves $P=0, R=0$.

The equations for $P_{1}, R_{1}$ are of the forms

$$
\begin{aligned}
& P_{1} \equiv \alpha_{1}^{(1)} y^{3}+\alpha_{0}^{(2)} y^{2}+\alpha_{1}^{(3)}+\alpha_{2}^{(2)}=0, \\
& R_{1} \equiv \beta_{0}^{(1)} y^{3}+\beta_{0}^{(1)} y^{2}+\beta_{0}^{(3)} y+\beta_{1}^{(3)}=0,
\end{aligned}
$$


where the coefficients $\alpha_{i}^{(j)}, \beta_{i}^{(j)}$ are polynomials in $x$ in which $j$ is the highest and $i$ the lowest power involved. Eliminating $y$ from these equations we obtain the determinant

$$
\Delta_{1} \equiv\left|\begin{array}{llllll}
\alpha_{1}^{(1)} & \alpha_{0}^{(2)} & \alpha_{1}^{(3)} & \alpha_{2}^{(2)} & 0 & 0 \\
0 & \alpha_{1}^{(1)} & \alpha_{0}^{(2)} & \alpha_{1}^{(3)} & \alpha_{2}^{(2)} & 0 \\
0 & 0 & \alpha_{1}^{(1)} & \alpha_{0}^{(2)} & \alpha_{1}^{(3)} & \alpha_{2}^{(2)} \\
\beta_{0}^{(1)} & \beta_{0}^{(1)} & \beta_{0}^{(3)} & \beta_{1}^{(3)} & 0 & 0 \\
0 & \beta_{0}^{(1)} & \beta_{0}^{(1)} & \beta_{0}^{(3)} & \beta_{1}^{(3)} & 0 \\
0 & 0 & \beta_{0}^{(1)} & \beta_{0}^{(1)} & \beta_{0}^{(3)} & \beta_{1}^{(3)}
\end{array}\right|=0 .
$$

This equation is of the fourteenth degree in $x$, the lowest term being of the second degree. Thus $x=0$ is a double root; the other twelve roots give the singular points $D, F, T, C, B_{1}, B_{2}, R_{1}, R_{2}, U_{1}, U_{2}, U_{3}, U_{4}$.

The equation $\Delta_{t}=0$ can be solved only when it is made numerical by giving particular values to the constants. The roots, which may be complex for general values of the coefficients, must first be separated and their values found by continued approximations. This procedure gives, indeed, the singular points required but leads to no criterion of admissibility. A more fruitful method is to trace the original curves $P_{1}=0, R_{1}=0$ for certain values of the elements, and then, varying these elements, to note what changes take place in the singular points both as to their positions and as to their admissibility.

The equation $R=0$ is homogeneous in $c, a$; consequently a variation of the ratio $c / a$ may be applied very easily. But in practice the initial choice of $c / a$ will usually be sufficient. In fact, the important terms in the perturbative development are those corresponding to small values of

$$
m_{1} \nu+m_{2} \nu^{\prime}
$$

where $\nu, \nu^{\prime}$ are the mean motions. We have approximately, for $n$ very large.

from which

$$
\iota \nu+c \nu^{\prime}=0,
$$

$$
\frac{c}{a}=-\frac{\nu}{\nu^{\prime}}=-|\beta|^{3} .
$$

The most important applications of the theory under discussion will be to the case of Jupiter and a small planet. The particular values of the elements are chosen in what follows with reference to this problem. Let $L_{1}^{2}, \sin \phi$, etc., be the elements as defined above of the orbit of one of the small planets and let $L^{\prime 2}, \sin \phi^{\prime}$, etc., be the elements of the orbit of Jupiter. Assume the value $\beta=2$, then the successive reductions of $-a / c$ become $\frac{1}{2}, \frac{1}{3}$, $\frac{5}{14}$, etc. This choice 
has the advantage of making all the singular points real in the case of zero inclination and coincident major axes, the necessary and sufficient condition being $c / a<0$. For the purpose of more accuracy in representing the curves $P=0$, $R=0$ by means of $P_{1}=0, R_{1}=0$ we shall assume $\tau, \tau^{\prime}, j$ somewhat smaller than in the case in nature just suggested. We assume accordingly that

$a=-1, \quad c=2, \quad|\beta|=2, \quad \tau=.03, \quad \tau^{\prime}=.01, \quad j=-.0005$.

If the perihelia coincide at the node, we have

if at $\pi / 2$ from the node,

$$
\beta=\beta_{0}=\gamma=\gamma_{0}=2
$$

$$
\beta=\beta_{0}=-\gamma=-\gamma_{0}=2 .
$$

On introducing these values, the equations $P_{1}=0, R_{1}=0$ become

$$
\begin{aligned}
& P_{1}=y^{3}[-2 x]+y^{2}\left[5 x^{2}-.02 x+.0009\right]+y\left[-2 x^{3}+.04 x^{2}-.0020 x \mp .0010 x\right] \\
&+.0004 x^{2}=0 . \\
& R_{1}=y^{3}[2 x+.06]+y^{2}[-.0036]+y\left[-2 x^{3}-.10 x^{2}+.0068 x \pm .0030 x\right. \\
&\quad-.000024 \mp .000030]+.04 x^{3}-.0008 x^{2}-.000016 x \neq .000020 x=0
\end{aligned}
$$

the double sign arising through $\gamma=\gamma_{0}= \pm 2$.

In the table on the following page are the equations resulting from particular values of $x$ near the origin. The roots of the equation are multiplied by 100 and the corresponding coefficients and roots are set down. In the first column the values of $x$ are given; the two single and two clouble columns following give the coefficients of $y^{3}, y^{2}, y$ and the constant term of the reduced equation. The three triple columns give the roots of the cubic in $y$. They represent respectively the roots for $j=0 ; j=-.0005, \gamma=\gamma_{0}=2 ; j=-.0005, \gamma=\gamma_{0}=-2$.

\section{\$4. The Criterion of Admissibility.}

It has been stated in $\S 1$ that for a singular point of $\Phi(z)$ to be admissible two singular points of $F(z, t)$ must have come into coincidence from opposite sides of the contour $|t|=1$. But $F(z, t)$ is a multiform function of $z, t$ of an infinite number of determinations. For $F(z, t)$ contains the expression $P$ uniform in $x, y$, which are in turn multiform in $z, t$, as is seen from the relations

$$
z=x^{a} e^{\frac{a \tau}{\sigma}\left(\frac{1}{x}-x\right)} y^{a} e^{\frac{c \tau^{\prime}}{\sigma^{\prime}}\left(\frac{1}{y}-y\right)}, \quad t=x^{\frac{1}{c}} e^{\frac{\tau}{\sigma \sigma}\left(\frac{1}{x}-x\right)} .
$$

The discussion for admissibility must then be made for each determination of $F(z, t)$, that is, upon each sheet of the corresponding Riemann surface. 

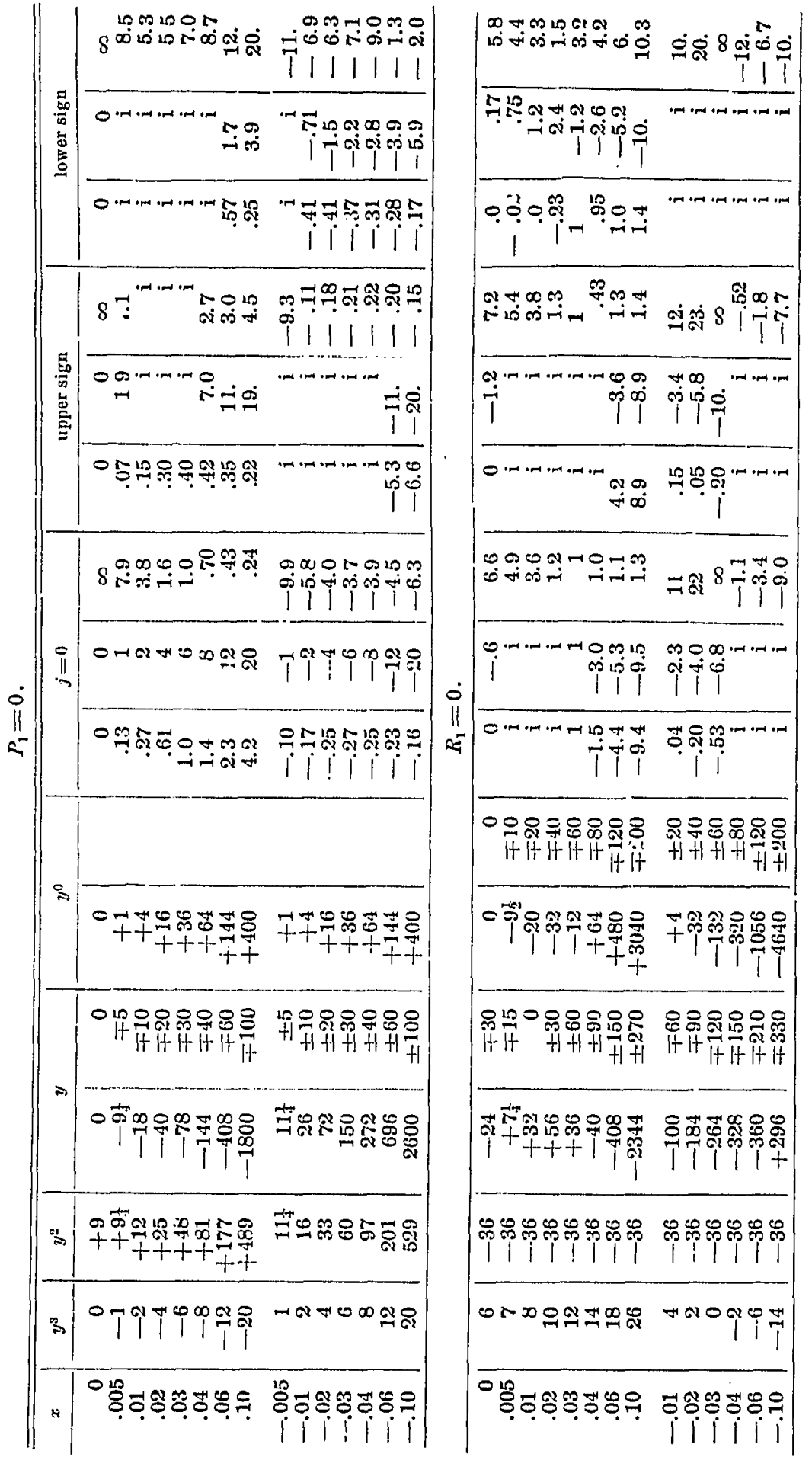
For the particular case $\tau^{\prime}=0$ PoIncare has overcome the difficulty. Using this, value of $\tau^{\prime}$ in $z$ above and solving for $y$, we get

$$
y=z^{\frac{1}{c}} x^{-\frac{a}{c}} e^{-\frac{a \tau}{c \sigma}\left(\frac{1}{x}-x\right)}
$$

in which it is seen that $y$ is uniform in $z^{1 / c}, x^{1 / c}, i$. e., in $z^{\prime}, x^{\prime}$ if we put

$$
x^{\frac{1}{c}}=x^{\prime}, \quad z^{\frac{1}{n}}=z^{\prime} \text {. }
$$

Also $P(x, y)$ is uniform in $z^{\prime}, x^{\prime}$, and in these variables $F(z, t)$ has only two determinations, namely those of the radical $P-\underline{1}$. This is effectively a reduction to one sheet, as these two determinations are equal in numerical value and opposite in sign. In the new variables the initial contour $|t|=1$ becomes $\left|x^{\prime}\right|=1$. This method has been extended by Coculesco to the case of small values of $\tau^{\prime}$, where as a first approximation the eccentric and mean anomalies are considered equal.

In general it can be shown by expanding $z^{\prime}$ as a power series in $\tau^{\prime}$, and neglecting terms above the $n$th power (as is permissible except for values of $y$ of the same order as $\tau^{\prime}$, such as occur along certain branches of the curve $P=0)$ that $F(z, t)$ becomes a function of multiplicity $2 n$ in the variables $z^{\prime}, x^{\prime}$. The geometrical meaning of this transformation as applied to the contomx $|t|=1$ is interesting, but the investigation is unnecessary to the present discussion. For it will be shown that the admissibility of points for the general case of $\tau^{\prime} \neq 0$ can be determined from the discussion of the case of $\tau^{\prime}=0$.

In fact, Porncare has shown that, in general, a singular point of $\Phi(z)$ cannot change its character as regards admissibility for any variation of the elements. The exceptional cases, as we shall see, are those for which there arises a change in the combinations of the singularities of $F(z, t)$ which form the singularities of $\Phi(z)$. We proceed to establish a theorem simpler in form than the original theorem by Porncars, but embodying its essential point. This theorem may be enunciated thus :

I. For the values $|z|=1$ the singular points of $F(z, t)$ cannot cross the circle $|t|=1$.

Having seen how the circle $|t|=1$ can be replaced by either $|x|=1$ or $\left|x^{\prime}\right|=1$, we are at liberty to make the discussion in the plane of $x$. Consider the relation

$$
\left.z=x^{a} e^{\frac{a \tau}{\sigma}\left(\frac{1}{x}-x\right)} y^{c} e^{c \tau^{\prime}} \frac{1}{y}-y\right) .
$$

If we let $z$ be a parameter, this relation is an implicit multiform function of the two complex variables $x$ and $y$. We have seen that it has an infinite number of determinations. There corresponds then to the function $z(x, y)$ a Riemann 
surface $S$ with an infinite number of sheets. The surface varies when the parameter $z$ is varied, and for $|z|=1$ becomes the surface $S_{0}$. Upon this surface trace the circle $C_{0}$ defined by

$$
|x|=|y|=1
$$

This is possible, for if $x$ be given a value of modulus unity then we can choose a value of $y$ having the modulus unity in such a manner that $z$ may have any value whatever of modulus unity. On the other hand if $|x|=|y|=1$ and $x=y$, then there corresponds a unique point on the circle of unit radius in the z-plane.

Now to each point on the Riemann surface $S$ there correspond definite values of $x$ and $y$, and consequently definite values of the eccentric anomalies $u$ and $u$. The points on the circles

$$
|x|=|y|=1
$$

give real values of the eccentric anomalies, i. e., a real point on each of the orbits. But in this case $F(z, t)$ has a singularity only when the distance between the two bodies is zero, that is, when

$$
P=0 ;
$$

or, when the distance of one of the planets from the sun is zero, that is, when

or

$$
1-\sin \phi \cos u=0 \text {, }
$$

$$
1-\sin \phi^{\prime} \cos \imath^{\prime}=0 \text {. }
$$

Excluding then the case of intersecting orbits and also the limiting case of the problem of two bodies where the orbit reduces to a straight line, it is impossible that for any variation of the elements a point on the circle $C_{0}$ shall become a singular point of $F(z, t)$. Therefore a singular point of $F(z, t)$ can not cross the circle $|t|=1$ for values $|z|=1$.

A singular point of $\Phi(z)$ has been defined as being admissible when it is formed by the coming into coincidence of two singularities of $F(z, t)$ from the interior and exterior respectively of the initial contour $|t|=1$. The theorem just proved may be applied to the question of the permanence in the character of admissibility of the singular points of $\Phi(z)$. To distinguish among these singular points certain definitions are useful. We assume that the elements of the orbits, with the exception of the anomalies, remain constant. Let $A, B$ be two singular points of $\Phi(z)$ at which $z^{\prime}$ has the values $z_{1}^{\prime}, z_{2}^{\prime}$ and assume $\left|z_{1}^{\prime}\right|<\left|z_{2}^{\prime}\right|<1$. When $z^{\prime}$ is made to vary starting from the point $A$, then the corresponding points in the plane of $x^{\prime}$ separate and move subject to the conditions defining singular points of $F(z, t)$. If in the plane of $x^{\prime}$ there is a 
means of direct passage from $A$ to $B$ along a line of continuously increasing modulus of $z$, then $B$ is subordinate to $A$.* $^{*}$ The means of passage is the locus furnished by one of the equations (1), (2), $P=0$.

If the point $z^{\prime}$ encounters no other singular points in its course, and if the points $A$ and $B$ are only double points, then $A$ gives three terminal values in the plane of $x^{\prime}$, one proceeding from $A$ directly and two proceeding from the branch separating at $B$.

We define a singular point as primary if it has no admissible subordinate point. It is secondary if it has an admissible subordinate point. A primary point may be either admissible or inadmissible; a secondary point is pseudoadmissible, for, although there correspond to it terminal points both within and without the circle $\left|x^{\prime}\right|=1$, there can be no change of elements which will bring it upon the circle of convergence $\left|z^{\prime}\right|=r^{\prime}$ without also causing it to pass through the circle of absolute value of its subordinate point, and the subordinate point itself determines the limit of convergence of the Laurent series.

With these definitions we proceed to the discussion of admissibility, taking up the different cases that may arise. It is requisite first to establish the theorem :

II. A primary singular point of $\Phi(z)$ can not change its character as regards admissibility for any variation of the elements, and this character can be determined by varying $z^{\prime}$ along any path whatever of increasing modulus.

Consider the method of formation of the singular points of $\Phi(z)$. The variables $x$ and $y$ satisfy certain equations of the series (1), (2), $P=0, R=0$. The intersections furnished by these equations give singular points of $\Phi(z)$ of the first and second species, and to such a point there corresponds a definite value of $z^{\prime}$, that is, a point in the plane of $z^{\prime}$. Instead of finding the intersections indicated and then computing the valnes of $z^{\prime}$, it is simpler to introduce certain auxiliary curves to take the place of (1), (2), $P=0, R=0$, whose intersections give the required singular points of $\Phi(z)$. Let

$$
\alpha(x, y)=0
$$

be any one of the curves (1), (2), $P=0, R=0$. We have

$$
z^{\prime}(x, y)=r^{\prime} .
$$

When $r^{\prime}$ varies in a given manner, the points of intersection of $\alpha=0$ and $z^{\prime}=r^{\prime}$ describe certain curves; in particular, if $z^{\prime}$ describes the circle

$$
\left|z^{\prime}\right|=r^{\prime}
$$

the singular points arising from the intersections of the curves will cause $x^{\prime}$ to

* Poincaré, loc. cit., p. 308. 
describe certain $\beta$-curves in its plane. The curves $\alpha=0$ may be replaced by the corresponding $\beta$-curves. Mere intersections of the $\beta$-curves are not singular points of $\Phi(z)$; the tracing points must coincide for some value of $z^{\prime}$.

For the initial circle $\left|z^{\prime}\right|=1$ the $\beta$-curves in the plane of $x^{\prime}$ do not intersect the circle $\left|x^{\prime}\right|=1$, by theorem $I$. Now let the circle $\left|z^{\prime}\right|=r^{\prime}$ contract, keeping the origin fixed. The $\beta$-curves sweep over certain portions of their plane. The significance of this is that corresponding to any continuous path whatever from any point on the outer circle $\left|z^{\prime}\right|=1$ to any point on the inner circle $\left|z^{\prime}\right|=r^{\prime}$, there are certain paths lying entirely within the region swept over by the $\beta$ curves. Suppose that when the circle $\left|z^{\prime}\right|=r^{\prime}$ is reached two tracing points of the $\beta$-curves coincide. Corresponding to this point in the $x^{\prime}$-plane is a point on the circle $\left|z^{\prime}\right|=r^{\prime}$, namely one of the singular points of $\Phi(z)$. Now reverse the variation of $z^{\prime}$, and starting with the singular point let $z^{\prime}$ go to a value $\left|z^{\prime}\right|=1$ along any path whatever consistent with the continuous increase of the radius of the circle on which it is found. The points in the $x^{\prime}$-plane accordingly trace definite loci in the swept-over area and rest finally on the initial $\beta$-curves corresponding to $\left|z^{\prime}\right|=1$. These curves being entirely within or entirely without the circle $\left|x^{\prime}\right|=1$, the terminal values of $x^{\prime}$ are definitely within or without the circle $\left|x^{\prime}\right|=1$ for any $z^{\prime}$-path to the circle $\left|z^{\prime}\right|=1$; whence we conclude that the point can not change its character as regards admissibility for any change of the elements, for this would necessitate that some of the points of the $\beta$-curve should cross the contour

$$
|x|=|y|=1
$$

which we have shown to be impossible.

If in a series of singnlar points, connected by relations of subordination as defined previously, the one first encountered is inadmissible, the circle $\left|z^{\prime}\right|=r^{\prime}$ may be contracted over this singular point, since a deformation of the contour in the plane of $x^{\prime}$ is not rendered impossible. This may be repeated until an admissible point is renched. It is evident that the theorem applies to this point as well as to all the preceding points of the series, that is, to all the primary points of the problem.

Consider now all the points both primary and secondary in the z-plane connected in any manner whatever as required by the definition of subordination and as permitted by the method of varying $z$. If the elements be varied continuously these singular points likewise vary continuously. Their curves of connection must be deformed if necessary to correspond to the displacements. It is proposed to account for the behavior and the consequent character of the points for all possible changes on their relative positions.

Let $A$ represent a typical singular point with $B$ as an immediate subordinate 
point, and consider any particular series of connected points. Let $B$ pass through $A$. Then :

(1) If both were originally inadmissible they remain so after the change, for their combined terminations in the plane are still on the same side of the unit circle.

(2) If $A$ were originally admissible and $B$ inadmissible, the passage of $B$ through $A$ leaves $A$ admissible and renders $B$ admissible and secondary, i. e., psendo-admissible.

(3) There is no case of $B$ admissible and $A$ inadmissible.

(4) If $B$ is admissible, then $A$ is pseudo-admissible, and the passage of $B$ through $A$ gives the following result:

(a) If $A$ were intrinsically admissible, that is, if its admissibility did not depend upon a branch of $B$ not common to $A$ and $B$, then $A$ remains admissible and $B$ becomes psendo-admissible.

(b) If $A$ were not intrinsically admissible, then $A$ ceases to be admissible, and $B$ remains admissible.

This discussion covers all cases of interaction of primary singular points. This interaction produces certain secondary points. If we consider the passage of a secondary admissibility through an immediate subordinate primary admissibility, we shall have one of the above four cases, consequently the converse results holld. In fact, these converse relations are contained in the cases as already stated. We have: (1) is its own converse, $(4)(a)$ is its own converse, (3) does not exist, (2) is the converse of (4) (b),

It has been assumed that $B$ actually passed through $A$, but the results would be the same if it passed by it simply, $i$. e., passed throngh it in absolute value.

It should be remarled here that the variation of $z$ from a secondary point to to $|:|=1$ is not entirely arbitrary. For if all subordinate points are not taken account of, it may easily happen that we get an apparent inadmissibility when the point is actually admissible as found by the path through the subordinate point.

This completes the theory of admissibility. Before proceeding to the discussion of the points found in $\$ 3$ it should be remarked that there are for each point in addition $c-1$ other's of the same modulus and differing in amplitude by the $\operatorname{arcs} k \pi / c$. Porncaré showed that these points are all admissible or inadmissible according as one of their number is admissible or inadmissible, and this holds true in the general case.

\section{\$5. Discussion of the Singular Points.}

Having seen that the singular points which arise in case of particular elements can not, as a rule, change their character as regards admissibility for a change of elements, it is advantageous to make use of the simpler cases to determine 
what points are admissible and to follow these points when certain of the elements are made to vary continuously, in order to determine in the general case the points that are admissible.

Accordingly we make direct use of the results of Porncare and Coculesco already cited. Let us designate three cases as follows: Case I, $j=0$; Case II, $j \neq 0$, perihelia coincide at a node ; Case III, $j \neq 0$, perihelia coincide at ninety degrees from a node. In the figures $1,2,3$ the light and heavy lines are the curves in the case $j=0, j \neq 0$ respectively, also the full lines are the curves $P_{1}=0, x=\tau, y=\tau^{\prime}$, and the broken lines are curves $R_{1}=0$. The

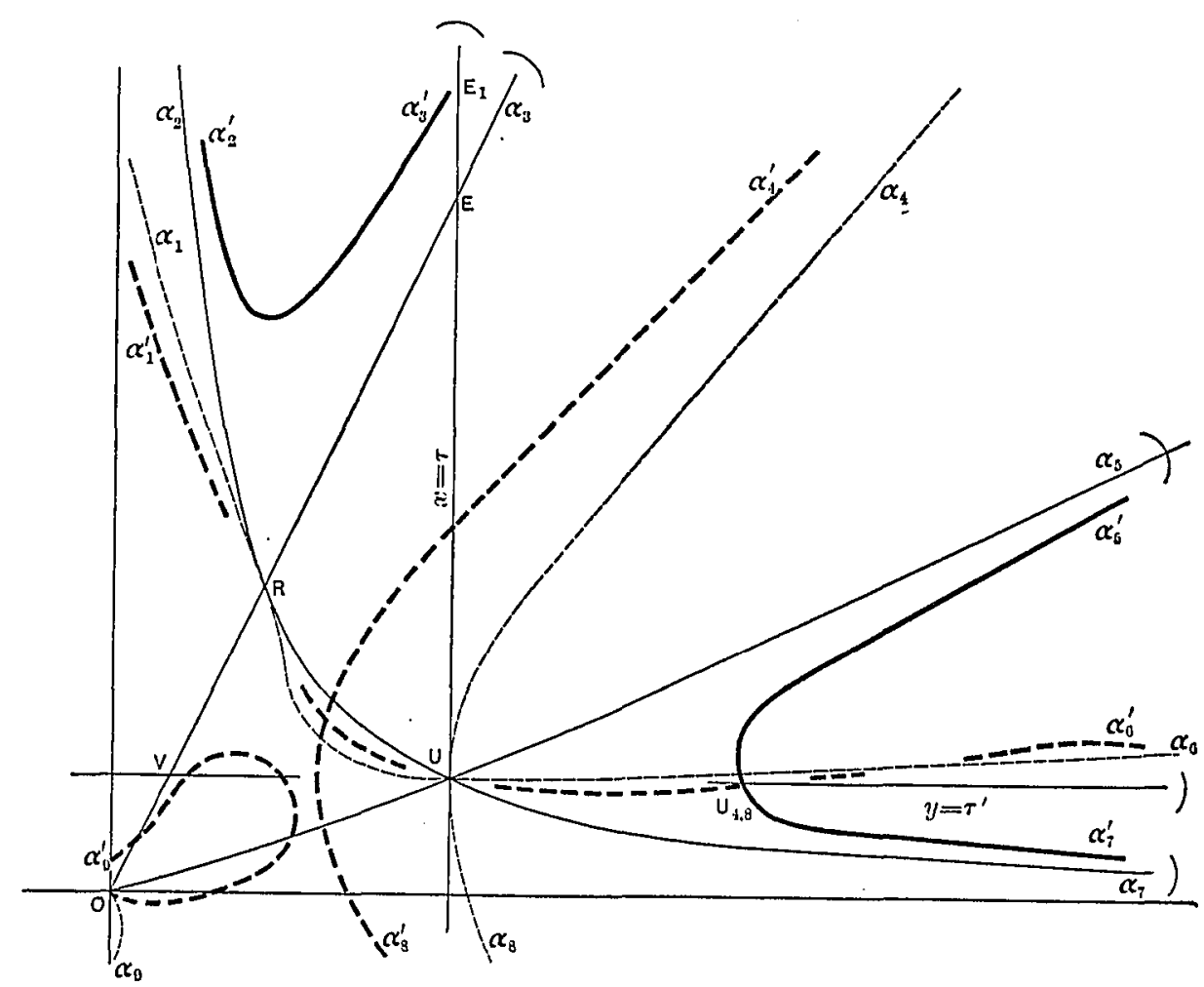

Fic. 1.

positions of the small arcs show where, with respect to the circle $\left|x^{\prime}\right|=1$, the points on the curves $P_{1}=0, x=\tau, y=\tau^{\prime}$ lie for which $\left|z^{\prime}\right|=1$.

Case I. $j=0, \bar{\omega}=\bar{\omega}^{\prime}$. This is the case discussed by PoIncsin and Coculesco. The singular points have alrendy been classified in $\S 2$. Of the points of the first species $B, R, E, W, X, Y$ are inadmissible and $U, V$ are admissible. Of the points of the second species, $C, F$ are inadmissible and $D$, $T$ are admissible.

The variation of $z$ is as follows: On the branches $\beta_{4} \alpha_{3}, z$ decreases to $C$, which in this case coincides with $B$, is a minimum at $C$, increases from $C$ to $O$, 
changes at $O$ from $|z|=\infty$ to $|z|=0$, and increases from $O$ through $E$. On the branches $\beta_{2} \beta_{6}, \alpha_{2} \alpha_{7}, z$ decreases to $D$, is a minimum at $D$, increases throngh ' $B$ to $P,|z|=\infty$ at $P,|z|=0$ at $Q$, increases to $F$, which in this case coincides with $R$, is a maximum at $F$, decreases from $F$ through $V$. On the branch $\beta_{1} \alpha_{5}, z$ increases to $T$, is a maximum at $T$, decreases from $T$ to $O$, changes at $O$ from $|z|=0$ to $|z|=\infty$, decreases from $O$ to $U$, and increases from $U$. Along $x=\tau$ the value of $z$ increases with $y$ positive, and along $y=\tau^{\prime}$ it increases with $x$ positive.

Case II. $j \neq 0, \bar{\omega}=\bar{\omega}^{\prime}=0$. The curves in this case are given in Figures 1,2 , which are plotted with the values given in Table I. The branches $\alpha_{1}, \alpha_{2}$.

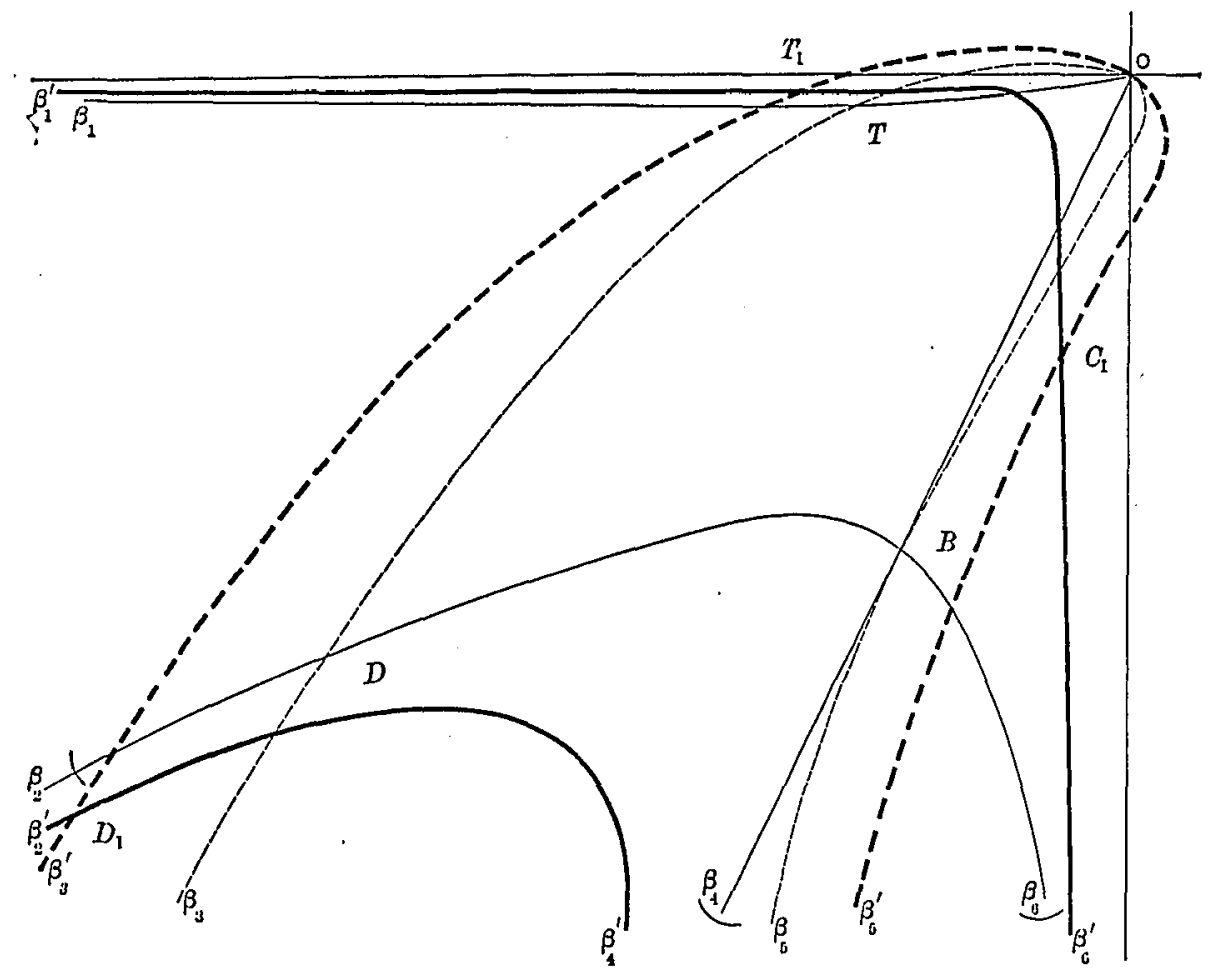

Fra. 2.

etc., are the curves of the Case $I$, just considered ; the branches $\alpha_{1}^{\prime}, \alpha_{2}^{\prime}$, etc., are for present consideration. The curves $P_{1}=0, x=\tau, y=\tau^{\prime}$ are represented in full lines, and the curves $R_{1}=0$ by dotted lines.

Consider the changes which the original curves undergo when the inclination is made to vary from zero, in other words when $j$ varies from zero to a small negative quantity. The segments $\alpha_{2} R$ and $R O$ vary continuously to form the branch $\alpha_{2}^{\prime} O$. The segments $\alpha_{3} R, R U, U \alpha_{5}$ form the branch $\alpha_{3}^{\prime} U_{2} \alpha_{i 5}^{\prime}$. The segments $O U, U \alpha_{7}$ form the branch $O U_{1} \alpha_{7}^{\prime}$. The straight lines $x=\tau, y=\tau^{\prime}$ 
are of course unchanged. The branches $\alpha_{1} \alpha_{0}$ and $\alpha_{1} \alpha_{8}$ become the branches $\alpha_{1}^{\prime} \alpha_{6}^{\prime}$ and $\alpha_{1}^{\prime} \alpha_{8}^{\prime}$ respectively.

The singular points for $j$ sufficiently small will lie very near those of Case I. The point $R$ is in reality a combination of what would be in general the points $F$ and $R$. This coincidence is due to the choice of constants in the particular numerical case computed. A change of the ratio $c / a$ is sufficient to separate the points $F$ and $R$ without changing the curve $P=0$ on which they lie. But these points can be separated otherwise. The branch $\alpha_{1} R U$ is tangent to the branch $\alpha_{2} R U$; this gives two coincident points of intersection at $R$. The branch $\alpha_{1} R U$ also cuts the branch $\alpha_{3} R O$ at $R$ in a point of the second species $R_{2}$. Thus $R$ is composed of the points $F, R_{1}, R_{2}$, all of the secoud species. When $j$ varies in the manner indicated the point $F^{\prime}$ remains real and takes a position $F_{1}$; the two points $R_{1}, R_{2}$ become conjugate imaginaries.

The point $U$ in the simple case is composed of nine points. These arise as follows: each of the two branches of $R=0$ which pass through $U$ cuts each of the branches of $P=0$ at the same point, giving rise to four points $U_{1}, U_{2}, U_{3}, U_{4}$ of the second species; each of the straight lines $x=\tau, y=\tau^{\prime}$ cuts each of the branches of $P=0$ in the same point, giving rise to four coincident points of the first species, $U_{5}, U_{6}, U_{7}, U_{8}$; the point $U$ is also the intersection of $x=\tau$, $y=\tau^{\prime}$. Now vary $j$ as before; the nine points all separate. $U_{1}$ and $U_{2}$ of the second species remain real. $U_{5}$ remains real. $U_{0}$ is imaginary in the figure, but it is evident that for sufficiently small values of $j$ it is real. In fact it leaves $U$ as a real point and different from the point $E$, these two points approaching each other along the line $a=\tau$. For a certain value of $j$ the points $E$ and $U_{6}$ coincide, and when $j$ is given still larger values they become conjugate imaginaries. The points $U_{3}, U_{4}$ are conjugate imaginaries; the points $U_{7}, U_{5}$ are also conjugate imaginaries.

The remaining points $V$, of the figure, and $W, Y, X$, not represented in the figure, take positions near their respective original positions.

Discussion. - By virtue of the theorem established in $\$ 4$ it is not necessary to discuss the points $E, F, R_{1}, R_{2}, V, W, X, Y$, since we have already determined the nature of the corresponding points in the simpler case. We have only to consider the nine points corresponding to $U$.

The equation $P=0$ is of the form

$$
P=S T+j A \text {. }
$$

We proceed to find the form of this curve for the values of $x$ lying between some value $x$ of the order $\tau$, and the value $x=1$. The product $S T$ considered as a polynomial in $y$ is of the fourth degree, while $A$ is a polynomial of the third degree in $y$. By talking $j$ sufficiently small we can make the curve

$$
S T+j A=0
$$


lie as close as we choose to the curve

$$
S T=0
$$

throughout the region under consideration; for the coefficient of the highest power in $y$ remains unchanged, and all the coefficients of $A$ are either finite or small.

Then for a given value of $x$ the corresponding values of $z^{\prime}$ along similar branches in the two cases will be approximately equal. In the case of $j=0$ the terminal values of $x^{\prime}$, when $z^{\prime}$ described the straight line of constant amplitude, were definitely within or without the circle $\left|x^{\prime}\right|=1$. It is possible in the present case to choose $j$ small enough to make the terminal value of $x^{\prime}$, relative to the circle $\left|x^{\prime}\right|=1$, correspond exactly to those in Case I. The character of the real points $U_{1}, U_{2}, U_{5}, U_{6}$ is now evident.

$U_{6}$ is formed by the intersection of $x=\tau$ with $\alpha_{3}^{\prime} U_{2} \alpha_{5}^{\prime}$. The two points on separation follow their respective branches in the direction of $y$ increasing. They reunite in $E$. But $E$ is inadmissible, therefore $U_{6}$ is inadmissible.

$U_{i,}$ is incadmissible. The two points which separate are found to follow $U_{5} O$ and $x=\tau$ in the direction of $y$ decreasing. The terminal values of $x$ are both without the circle $\left|x^{\prime}\right|=1$.

$U_{1}$ gives $z^{\prime}$ a minimum. When $z^{\prime}$ varies along the positive part of the real axis of its plane toward the value $z^{\prime}=1$, the two points $x^{\prime}$ on separation move along $U_{1} O$ and $U_{1} a_{7}^{\prime}$ respectively. The latter encounters in its course the point $U_{s}$. All the terminations for $U_{s}$ have been found to lie within $\left|x^{\prime}\right|=1$. The same is true for the terminations on the branch $U_{1} \alpha_{7}^{\prime}$, therefore $U_{1}$ is inadmissible.

$U_{2}$ is rulmissible. Since at this point $z^{\prime}$ is a minimum the values of $x^{\prime}$ on separation follow the branches $U_{2} \alpha_{3}^{\prime}$ and $U_{2} \alpha_{i 1}^{\prime}$. When $z^{\prime}$ attains the value unity we have for the first point $x^{\prime}-1$ and for the second $x^{\prime}>1$.

The points $U_{3}, U_{4}, U_{7}, U_{8}$ are left for considerntion. These points are all imaginary but their amplitudes are very small when $j$ is put small. Corresponding to each of these points the value of $z^{\prime}$ is imaginary with a small amplitude. Instead of varying $z^{\prime}$ along a line of constant amplitude, as was done in the case of the real points, let us vary $z^{\prime}$ along the straight line limited by the points $z_{0}^{\prime}$ and $z^{\prime}=1$, where $z_{i}^{\prime}$ is the value of $z^{\prime}$ at one of the singular points. The loci of the singular points which separate when $z^{\prime}$ is made to vary from the value $z_{0}^{\prime}$ are certain curves satisfying the relations

$$
\approx(x, y)=\zeta, \quad P(x, y)=0,
$$

for points of the second species $U_{3}, U_{4}$; or

Trans. Am. Muth. Soc. 33

$$
\approx(x, y)=\zeta, \quad y-\tau^{\prime}=0
$$


for points of the second species, $U_{7}, U_{8}$, where $\zeta$ is a parameter varying along the straight line limited by $z_{0}^{\prime}$ and $z^{\prime}=1$. In the case of $j=0$ this variation of $\zeta$ is along the real axis, and consequently the singular points on separation follow $P(x, y)=0$ in the real plane. For $j$ very small the singular points will describe curves lying not in the real plane, but very near the real part of $P=0$. The approximation becomes closer as $\zeta$ approaches $\zeta=1$, for which value the singular points have positions on the real part of $P=0$ not far from those terminal positions found for $j=0$.

Consider the singular points of the second species, $U_{3}, U_{4}$, formed by the intersections of the curves $P=0$ and $R=0$ in the neighborhood of the point $U$. Such an intersection implies that $z^{\prime}$ is a maximum or a minimum. In each case we know it to be a minimum. Then the four points $x^{\prime}$ after separating, two from $U_{3}$ and two from $U_{4}$, follow each of them one of the four segments of $P=0$, which approach coincidence with $U O, U R, U \alpha_{5}, U \alpha_{7}$, respectively.

An examination of the order of interchange of the branches near $U$ is necessary. But this discussion is practically obviated through a priori considerations. In fact, $U$ represents an ordinary node of the function $P=0$ in the case of $j=0$. When $j$ varies the interchange may take place in one of four different ways. The double point may continue to exist near $U$, or the two branches may cease to intersect and the continuous branches may lie near the original curve in any of three ways as follows : $\alpha_{5}^{\prime \prime} U R$ and $\alpha_{7}^{\prime \prime} U O, \alpha_{5}^{\prime \prime} U \alpha_{7}^{\prime \prime}$ and $R U O, \alpha_{i}^{\prime \prime} U O$ and $\alpha_{7}^{\prime \prime} U R$. It can easily be shown that if the node continued to exist for variations of $j$, there would be one or three admissible singular points according as one of the two points of the second species is or is not on the segment $U_{10} \alpha_{i j}^{\prime \prime}$. But it can be shown that for general values of $j$ there is no node $U_{10}$. Consider the three other possibilities. By virtue of the continuity of the function $P$ we see that each of the four segments which the singular points describe after separation is involved once and only once. We know that of the four terminal values of $x^{\prime}$ three are within the circle $\left|x^{\prime}\right|=1$ and one (on the branch $\alpha_{5}^{\prime \prime}$ ) is without this circle. Thus of the two conjugate imaginary points, $U_{3}, U_{4}$, one is admissible and one is inadmissible. This distinction will be considered later.

The points $U_{7}, U_{8}$ which are the imaginary intersections of $y=\tau^{\prime}$ and $P=0$ in the neighborhood of $U$ become real when $\bar{\omega}=\bar{\omega}^{\prime}=\pi / 2$, and are reserved for discussion under Case III.

The point $U$, which is the intersection of $x=\tau$ and $y=\tau^{\prime}$, is or is not admissible according as one of the points $U_{7}, U_{8}$, supposed subordinate, is or is not admissible. This illustrates a distinction made in the foregoing section. In fact, if the points on separation were permitted to follow the line $y=\tau^{\prime}$ only in the real plane, we should be led to conclude that $U$ is necessarily inadmissible. 
The curves as they exist in the third quadrant are represented by Fig. 2. The branches $\beta_{1}, \beta_{2}$, etc., are the curves of Case I, while $\beta_{1}^{\prime}, \beta_{2}^{\prime}$, etc., are the curves in the present case. From the figure it is seen that as $j$ varies from zero, the line $\beta_{1} T O B \beta_{6}$ becomes $\beta_{1}^{\prime} \beta_{6,}^{\prime}$, and $\beta_{2} D B \beta_{4}$ becomes $\beta_{9}^{\prime} \beta_{4}^{\prime}$. The branch $\beta_{3} D T O B \beta_{4}$ becomes $\beta_{3}^{\prime} O \beta_{4}^{\prime}$. At $B$ three points coincide. These separate when $j$ is made to vary, the point $C$ remaining real, $B_{1}$ and $B_{2}$ becoming conjugate imaginaries. The points $D, T$, remain real and their abscissas increase in absolute value.

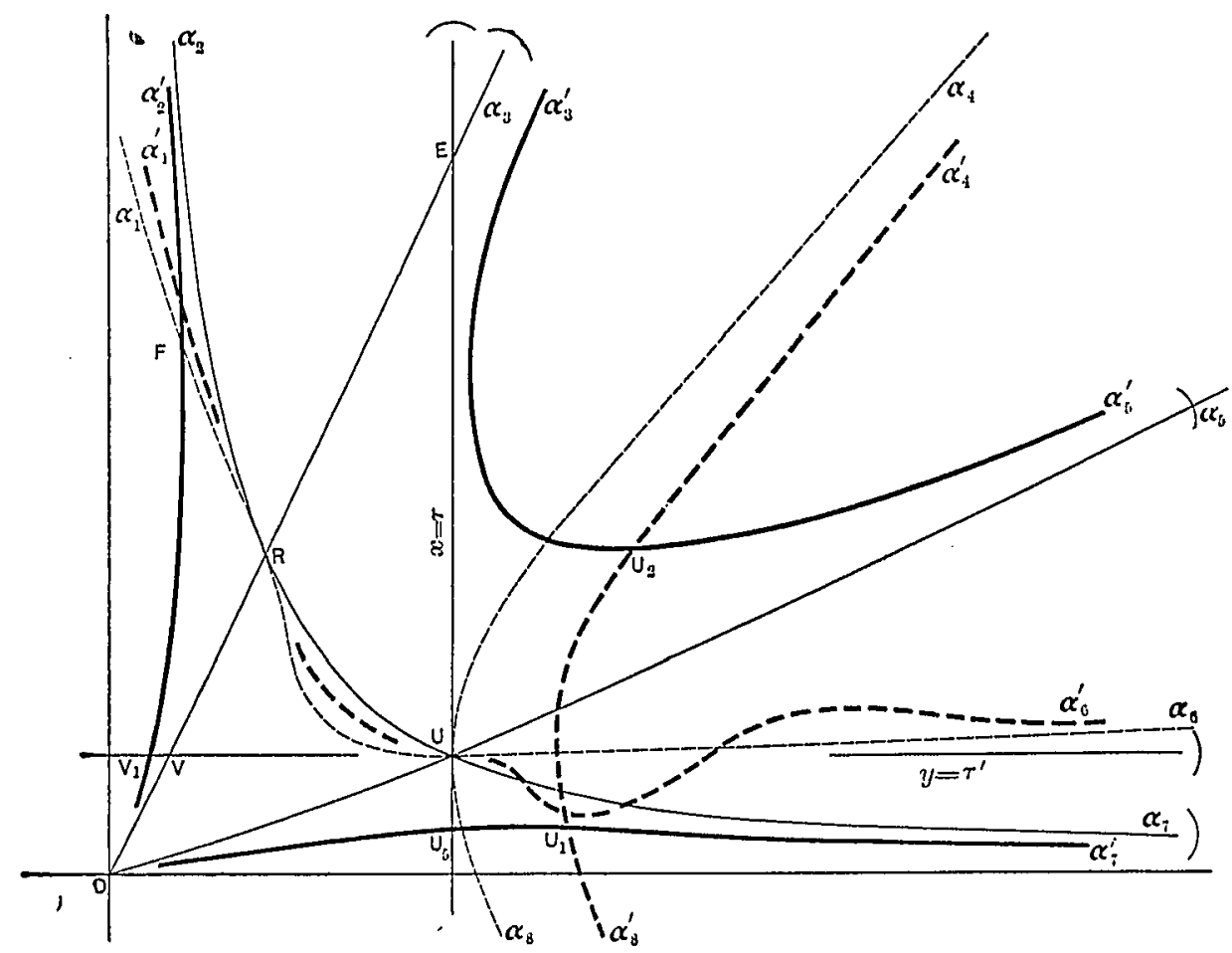

FIG. 3.

The points $B_{1}, B_{2}, C$ are inadmissible and the points $D, T$ are admissible, as was found in Case $I$.

Case III. $j \neq 0, \bar{\omega}=\bar{\omega}^{\prime}=\pi / 2$. In this case the curves as they exist in the first quadrant are represented by Fig. 3. They are plotted with the particular values given in Table I. The branches $\alpha_{1}, \alpha_{2}$, etc., are the curves in the case $j=0$; the branches $\alpha_{1}^{\prime}, \alpha_{2}^{\prime}$, etc., are for present consideration.

When $j$ varies from zero to the small value used in the table, the original curves vary as follows: $\alpha_{2} R \alpha_{3}$ becomes $\alpha_{2}^{\prime} \alpha_{3}^{\prime}$;ORUO remains a closed curve and recedes toward the point $O ; \alpha_{5} U \alpha_{7}$ becomes $\alpha_{5}^{\prime} \alpha_{7}^{\prime}$; the branches $\alpha_{1} R U \alpha_{6}$ 
and $\alpha_{4} U \alpha_{8}$ become respectively the branches $\alpha_{1}^{\prime} R U \alpha_{i j}^{\prime}$ and $\alpha_{4}^{\prime} \alpha_{8}^{\prime}$; finally, the branch $\alpha_{9} O$ passes through a loop form, then to the position $O \alpha_{9}^{\prime}$. The order of the separation of the branches here is seen to be opposite to that of Case II.

The singular point at $R$ breaks up into the points $F, R_{1}, R_{2}$. The points $F, R_{1}$ are conjugate imaginaries. $R_{2}$, the point lying below $R$, is real at first, but for the value of $j$ employed in the figure it has become imaginary. When the loop formed by the segments ORUO begins to recede toward the origin, the two points of intersection of this loop with a line $R U$, little different from the lower of the dotted lines $R U$, will be real and different. Designate by $U_{3}$ that one of the points near $U$. The value of $j$ continuing its variation, the points $U_{3}$ and $R_{2}$ come into coincidence and then separate to become conjugate imaginaries. Consider the variation of $z^{\prime}$ on this loop when $U_{3}$ and $R_{2}$ are real. The loop is cut by the line $y=\tau^{\prime}$ at the points $V$ and $U_{7}$. The variation of $z^{\prime}$ is as follows: zero at $O ; O V R_{2}$, increases; maximum, at $R_{2}$; $R_{2} U_{3}$, decreases; minimum at $U_{3} ; U_{3} U_{7} O$, increases ; infinity at $O$. Now let the loop decrease, the maximum point $R_{2}$ comes to coincide with the minimum point $U_{3}$, then $z^{\prime}$ continuously increases around the loop $O V_{1} U_{7} O$. The loop continuing to decrease, $V_{1}$ and $V_{7}$ appronch each other, coincide, and then become conjugate imaginaries. The curve $\alpha_{1}^{\prime} U \alpha_{i}^{\prime}$ and the line $y=\tau^{\prime}$ cut the curve $P=O$ at approximately the same point in the figure. The straight line $x=\tau$ cuts the curve $P=O$ in two imaginary points $U_{5}, U_{6}$.

Discussion. - By using the results of the two preceding cases the character of the singular points in the present case is easily determined.

$F$ is inadmissible.

$E$ is inadmissible.

$R_{1}$ has $F$ and $E$ as subordinate points and is itself inadmissible.

$R_{2}$ is a maximum point of $z^{\prime}$, and when $z^{\prime}$ increases the two points become conjugate imaginaries at first and then coincide at $R_{1}$. Thus $R_{2}$ is inadmissible.

$U_{t}$, the intersection of $\alpha_{5}^{\prime} \alpha_{i}^{\prime}$ and $U \alpha_{i i}^{\prime}$, is admissible. For, after separation, the two values of $x^{\prime}$ follow the branches $\alpha_{i,}^{\prime}$ and $\alpha_{i}^{\prime}$ respectively, the terminal on the former branch being without and that on the latter branch being within the circle $\left|x^{\prime}\right|=1$.

$U_{3}$, subordinate to $U_{t}^{r}$ for sufficiently small valnes of $j$, is admissible. The tangent to the curve $U \alpha_{6}^{\prime}$ at $U$ is negative thus giving $U_{8}$ a position above $U_{4}$. The terminal value of $x^{\prime}$ in the directions of $x$ increasing and $\alpha_{j}^{\prime}$ are respectively within and without the circles $\left|x^{\prime}\right|=1$.

$U$ has $U_{8}$ as a subordinate point and is consequently admissible.

$U_{7}$ is admissible; of its two branches one follows the curve $U_{i}, O$ and the other passes through $U$ and $U_{s}$ which are admissible.

$U_{3}$ is admissible; it has $R_{2}$ and $U_{7}$ as subordinate points, of which the latter is admissible. 

alone.

$V_{1}$ is admissible; it has at first $R_{2}$ and $U_{7}$ as subordinate points, and later $U_{7}$

$U_{5}$ and $U_{7}$, the intersections of $x=\tau$ with $P=O$ are admissible and inadmissible respectively. For, of the four branches $U R, U O, U \alpha_{7}, U \alpha_{5}$, only the latter gives a terminal value of $x^{\prime}$ greater than unity. With the argument used for $U_{3}$ and $U_{4}$ of Case II, the conclusion is evident. The discussion for the third quadrant is not difficult and may be omitted.

\section{$\S 6$. The Case of $\tau^{\prime}=0, j \neq 0$. Conclusion.}

Before passing to conclusions relative to the general case, it is interesting to consider what simplifications arise on taking the eccentricity of the orbit of the second planet zero, the eccentricity of the orbit of the first being small, and the mutual inclination small.

Neglecting in the equation $P=0$ the terms in $\tau^{\prime}$ and arranging in powers of $y$, we have

$$
\begin{aligned}
y^{2}\left[\gamma \sigma j x(x-\tau)^{2}-\beta \sigma x(1-x \tau)^{2}(1+j)\right]+y\left[(x-\tau)^{2}(1-x \tau)^{2}+\beta \beta_{0} \sigma^{2} x^{2}\right] & \\
& \left.+\gamma_{0} \sigma j x(1-a \tau)^{2}-\beta_{0} \sigma x(x-\tau)^{2}(1+j)\right]=0 .
\end{aligned}
$$

An approximate expression for this curve in the neighborhood of the origin can be obtained in a very much rednced form by neglecting all but terms of relatively low order in the various coefficients. Thus considering $x$ of the same order as $\tau$, and neglecting all but terms of the second order in the coefficients, there results the equation

$$
P_{2} \equiv y^{2}[-\beta \sigma x(1+j)]+y\left[(x-\tau)^{2}+\beta \beta_{0} \sigma^{2} x^{2}\right]+\gamma_{0} \sigma j x=0 .
$$

Likewise the equation $R=0$ becomes

$$
\begin{aligned}
R_{2} \equiv & y^{2}[a \beta(x-\tau)(1+j)+c \beta \sigma x(1+j)]+y[2 c \tau(x-\tau)] \\
& +\left[-a \beta_{0}(x-\tau)^{3}-c \beta_{0} \sigma x\left(x^{2}-\tau^{2}\right)+j\left\{-c \gamma_{0} \sigma x+a \gamma_{0}(x-\tau)\right\}\right]=0 .
\end{aligned}
$$

The eliminant $\Delta_{2}=0$ of $P_{2}=0$ and $R_{2}=0$ may be found as in $\$ 3$. It has for its roots the abscissas of points corresponding to $U, F, R_{1}, R_{2}, D, C, B_{1}, B_{2}$.

Equations $P_{2}=0, R_{2}=0$ may be written in the expanded forms

$$
\begin{aligned}
& P_{2} \equiv y^{2}\left[s_{11} x\right]+y\left[s_{22} x^{2}+s_{21} x+s_{20}\right]+\left[s_{33} x^{3}+s_{32} x^{2}+s_{31}^{\prime} x\right]=0, \\
& R_{2} \equiv y^{2}\left[t_{11} x+t_{10}\right]+y\left[t_{21} x+t_{20}\right]+\left[t_{33} x^{3}+t_{32} x^{2}+t_{31}^{\prime} x+t_{30}^{\prime}\right]=0,
\end{aligned}
$$

where $s_{31}^{\prime}, t_{31}^{\prime}, t_{30}^{\prime}$ are so designated because they contain all the terms in $j$, and 
to these terms the double sign is attached. Then $\Delta_{2}$ becomes

$$
\begin{aligned}
& {\left[s_{33} x^{3}+s_{32} x^{2}+s_{31}^{\prime} x\right]\left[u_{5} x^{5}+u^{4} x^{4}+\cdots+u_{0}\right]} \\
& +\left[t_{33} x^{3}+t_{38} x^{2}+t_{31}^{\prime} x+t_{30}^{\prime}\right]\left[v_{5} x^{5}+\cdots+v_{0}\right]=0,
\end{aligned}
$$

where the $u, v$ are functions of the $s, t$ as indicated. Or again,

$$
\Delta_{i 2} \equiv \lambda_{8} x^{s}+\lambda_{\tau} x^{\tau}+\cdots+\lambda_{\theta}=0 .
$$

Assuming the approximate values of the roots as found by plotting, they may be corrected by applying the well known formula

$$
x=x_{0}-\frac{\Delta_{2}\left(x_{0}\right)}{\Delta_{2}^{\prime}\left(x_{0}\right)},
$$

which is true for all simple roots, real or imaginary. The work may be reduced in the two cases under consideration by finding the four real roots first and then reducing the order of the equations.

In the two cases of $\bar{\omega}=\bar{\omega}^{\prime}=0$ and $\bar{\omega}=\bar{\omega}^{\prime}=\pi / 2$, the roots of $\Delta_{2}$ may be calculated very rapilly by the above formula when $j$ is varied little by little, since changes in $j$ will affect $s_{31}^{\prime}, t_{31}^{\prime}, t_{30}^{\prime}$ and $u_{i}, v_{i}$ in an alditive manner simply.

Since the choice of $\beta, \tau$ can be made once for all, and since $j$ can be made to assume any assigned value in the manner indicated, there remains for consideration the variation of the longitudes of the perihelia. Having taken $\tau^{\prime}=0$, we have $\bar{\omega}=\bar{\omega}^{\prime}$ and

$$
\beta=\beta_{0}=\frac{L^{\prime 2}}{L^{2}}, \quad \gamma_{0}=\frac{L^{\prime 2}}{L^{2}} e^{-2 i \bar{\omega}}
$$

When $\bar{\omega}$ is taken different from zero, imaginary terms affect the expressions $\lambda_{i}$. The roots of $\Delta_{2}=0$ become imaginary. They may be computed by the above formula as $\bar{\omega}$ varies from zero. The comprtation of $x_{i j}^{n}$ in the functions $\Delta_{2}\left(x_{0}\right), \Delta \nu_{1}$ is best effected by the use of trigonometric tables by writing

$$
x_{0}^{n}=\left|x_{0}\right|^{n} e^{i n \theta}=\left|x_{0}\right|^{\prime \prime}(\cos n \theta+i \sin n \theta) .
$$

From the foregoing case the means of extension to the most general case is now evident. We have seen that it is necessary to take account of the analytic possibility that a singular point may become admissible or cease to be admissible, but slight attention to the geometry of the variation of the singular points as the elements vary will suffice to guard against error. Probably the selection of the primary admissible points, as well as their tracing in the $x$-plane, with the application of the principle of the limits of the roots of an equation, will answer in most of the practical applications. Approximations may usually be made in the manner employed here, and where necessary recourse may be had to the original equations of a higher degree. 
Only points within the circle $|z|=1$ bave been considered in the foregoing discussion. After we have determined the coördinates $x, y$ of the singular point which gives $z$ nearest the circle, the point outside the circle $|x|=1$ and nearest it is found as follows. We have

$$
\Delta(x, i) \equiv X \Delta\left(\frac{1}{x},-i\right)=0,
$$

where $X$ is a power of $x$ and $\Delta$ is the equation in $x$ determining singular points. This shows that $1 / x$ is also a root of $\Delta$ if $-i$ is put for $i$. But this is effectively a change of elements, namely an interchange of $\beta$ with $\beta_{0}$ and of $\gamma$ with $\gamma_{0}$. Make this change, then

$$
\Delta(x,-i) \equiv X \Delta\left(\frac{1}{x}, i\right)=0 .
$$

Thus we see that by putting $-i$ for $i$ the lmowledge of the inner point which conforms to the demands of the problem enables us to find the required outer point.

The simplest relations from which to begin the variation of the perihelia are

$$
\bar{\omega}=0, \underset{2}{\pi}, \pi, \begin{aligned}
& 3 \pi \\
& 2
\end{aligned}
$$

with $\bar{\omega}$ coincident or opposite, which gives

$$
\beta\left(=\beta_{0}\right)= \pm \gamma\left(=\gamma_{11}\right)= \pm \frac{L^{\prime 2}}{L^{i}} .
$$

The variation of $c$ and $a$ has not been considered. These constants enter the equation $R=0$ only. This equation is homogeneous in $c$, $a$, so that if it is decided to vary the watio $c / a$ after a singular point is found, a factor may be entered rendily in each term. Otherwise the problem may be begun anew for each choice of $c / a, c$ and $a$ being small integers.

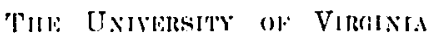
JTNE 1907 . 